\title{
Metabolomic Elucidation of the Effect of Sucrose on the Secondary Metabolite Profiles in Melissa officinalis by Ultraperformance Liquid Chromatography-Mass Spectrometry
}

Sooah Kim, Jungyeon Kim, Nahyun Kim, Dongho Lee, Hojoung Lee, Dong-Yup Lee,* and Kyoung Heon Kim*

Cite This: ACS Omega 2020, 5, 33186-33195

Read Online

ABSTRACT: Sucrose induces flavonoid accumulation in plants as a defense mechanism against various stresses. However, the relationship between the biosynthesis of flavonoids as secondary metabolites and sucrose levels remains unknown. To understand the change in flavonoid biosynthesis by sucrose, we conducted secondary metabolite profiling in Melissa officinalis treated with different levels of sucrose using ultraperformance liquid chromatography/quadrupole time-of-flight mass spectrometry. The partial least squares-discriminant and hierarchical clustering analyses showed significant differences in secondary metabolite profiles in M. officinalis at 50,150, and $300 \mathrm{mM}$ sucrose levels. The levels of 3 flavonoids such as quercetin $3-O-\beta$-D-glucosyl- $(1 \rightarrow 2)-\beta$-D-glucoside, 6-methoxyaromadendrin 3-O-acetate, and 3-hydroxycoumarin
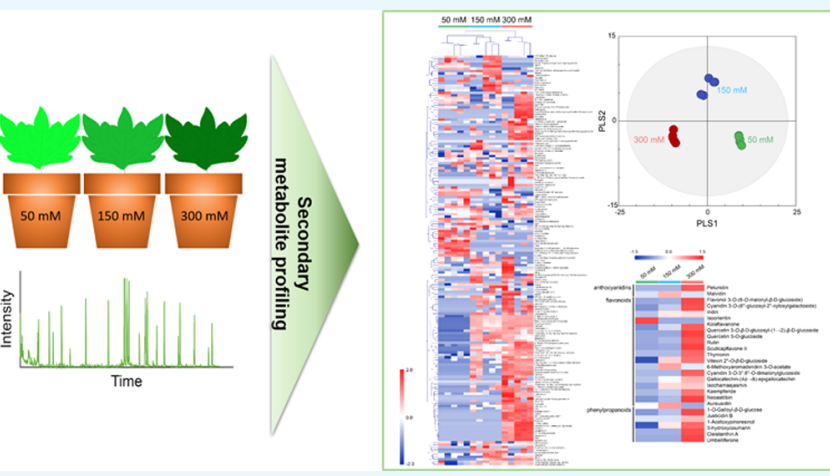
and 19 flavonoids including 6-methoxyaromadendrin 3-O-acetate, aureusidin, iridin, flavonol 3-O-(6-O-malonyl- $\beta$-D-glucoside) quercetin 3-O-glucoside, and rutin increased at 150 and $300 \mathrm{mM}$ sucrose, respectively, compared to $50 \mathrm{mM}$ sucrose, indicating that the flavonoids were accumulated in M. officinalis by a higher concentration of sucrose. This is the first investigation of the change in individual flavonoids as secondary metabolites in $M$. officinalis by varying sucrose levels, and the results demonstrate that the sucrose causes the accumulation of certain flavonoids as a defense mechanism against osmotic stress.

\section{INTRODUCTION}

Plants contain a variety of primary and secondary metabolites, which are the intermediate or end products of cellular processes. ${ }^{1}$ Secondary metabolites including flavonoids play an important role in various biochemistry and physiological processes in plants. Their levels are considered important because they are used in obtaining valuable information such as the physiological state; they reflect specific biochemical processes in plants as metabolite levels serve as the ultimate response of biological systems to various genetic or environmental changes. ${ }^{2}$

Metabolomics, the study of chemical processes involving the entire metabolome of an organism, is a useful tool in determining metabolites in response to such changes. Various analytical tools have been used for metabolite profiling of plants, including gas chromatography/mass spectrometry, liquid chromatography-mass spectrometry (LC-MS), ${ }^{5,6}$ and nuclear magnetic resonance. ${ }^{7,8} \mathrm{LC}-\mathrm{MS}$ is the most commonly used in secondary metabolite profiling of plants because it offers high selectivity and sensitivity and allows the analysis of nonvolatile, unstable, and high-molecular-weight compounds without derivatization. ${ }^{9,10}$
Melissa officinalis, a perennial herb distributed throughout East Asia, has been well known as a traditional medicine used in treating human disorders such as headache, digestion disorder, Alzheimer's disease, and cancer. ${ }^{11,12}$ Various secondary metabolites in $M$. officinalis are known to be responsible for antioxidative, antibacterial, anti-inflammatory, antifungal, and antitumor activities. ${ }^{13-16}$ Thus, many studies have manipulated the metabolism of $M$. officinalis to produce target secondary metabolites that can be used as valuable substances. $^{17,18}$

Sucrose can function as the hormone-like signaling molecule and control various metabolisms and growth in plants. ${ }^{19}$ It is an important factor affecting the synthesis of the secondary metabolites pathway including flavonoid. ${ }^{20,21}$ Secondary metabolites are well known to accumulate during stressful

Received: October 5, 2020

Accepted: December 2, 2020

Published: December 15, 2020 
Table 1. Identified Secondary Metabolites from M. officinalis with Retention Time and $\mathrm{m} / z$

\begin{tabular}{|c|c|c|c|c|}
\hline ESI & exact mass & mass error $(\mathrm{ppm})$ & matched metabolite from database & PubChem ID \\
\hline negative & 242.080 & 19.531 & lumichrome & 5326566 \\
\hline negative & 174.100 & 11.809 & $N^{5}$-ethyl-L-glutamine & 439378 \\
\hline negative & 258.062 & 4.088 & streptamine phosphate & 439934 \\
\hline negative & 276.025 & 2.137 & 2-carboxy-D-arabinitol 1-phosphate & 129417 \\
\hline negative & 232.016 & 0.655 & $N$-phosphohypotaurocyamine & 16019959 \\
\hline negative & 192.063 & 10.309 & valiolone & 443630 \\
\hline negative & 634.132 & 2.383 & actinorhodine & 441143 \\
\hline negative & 105.033 & 6.208 & cyanopyrazine & 73172 \\
\hline negative & 588.127 & 5.164 & kolaflavanone & 155169 \\
\hline negative & 184.999 & 15.551 & L-serine $O$-sulfate & 164701 \\
\hline negative & 278.115 & 9.500 & isohelenol & 15558 \\
\hline negative & 332.074 & 19.246 & 1-O-galloyl- $\beta$-D-glucose & 124021 \\
\hline negative & 331.082 & 3.990 & malvidin & 159287 \\
\hline negative & 504.169 & 7.928 & cellotriose & 5287993 \\
\hline negative & 128.047 & 0.992 & 2-hydroxy-cis-hex-2,4-dienoate & 11953951 \\
\hline negative & 317.066 & 7.140 & petunidin & 73386 \\
\hline negative & 110.037 & 8.061 & catechol & 289 \\
\hline negative & 514.115 & 1.270 & MK 571 & 5281888 \\
\hline negative & 650.252 & 14.857 & BQ 518 & 443291 \\
\hline negative & 292.121 & 17.599 & INF271 & 443080 \\
\hline negative & 856.254 & 4.466 & 7-hydroxylpradimicin A & 441176 \\
\hline negative & 342.110 & 14.691 & 3-(4-methoxyphenyl)-5,6,7-trimethoxy-4H-1-benzopyran-4-one & 248269 \\
\hline negative & 674.221 & 18.819 & premithramycin $\mathrm{A}^{\prime}$ & 443797 \\
\hline negative & 326.121 & 8.104 & robinobiose & 441428 \\
\hline negative & 490.171 & 19.730 & BMS-268770 & 56928083 \\
\hline negative & 344.108 & 12.008 & TRAM-34 & 656734 \\
\hline negative & 328.102 & 16.053 & 7-hydroxy-6-methyl-8-ribityl lumazine & 440869 \\
\hline negative & 518.159 & 0.550 & esmeraldic acid & 443632 \\
\hline negative & 216.027 & 4.060 & 5-carboxymethyl-2-hydroxymuconate & 54675765 \\
\hline negative & 192.047 & 6.535 & 6-(allylthio)purine & 3633259 \\
\hline negative & 133.038 & 11.861 & L-aspartate & 5960 \\
\hline negative & 405.100 & 5.991 & cefaloglycin & 19150 \\
\hline negative & 356.098 & 17.147 & Bay-K-8644 & 2303 \\
\hline negative & 168.019 & 12.028 & butanoylphosphate & 266 \\
\hline negative & 307.069 & 14.055 & narciclasine & 72376 \\
\hline negative & 150.032 & 1.013 & $\alpha$-oxo-benzeneacetic acid & 11915 \\
\hline negative & 471.150 & 4.355 & 10-formyldihydrofolate & 135398690 \\
\hline negative & 194.063 & 1.680 & 6-(isopropylthio)purine & 3698120 \\
\hline negative & 273.086 & 10.110 & brugine & 442998 \\
\hline negative & 296.105 & 18.929 & calophyllin B & 5281624 \\
\hline negative & 109.900 & 6.524 & calcium chloride anhydrous & 5284359 \\
\hline negative & 296.092 & 19.494 & 2,3,9,10-tetrahydroxyberberine & 443768 \\
\hline negative & 332.069 & 10.829 & hypoxylone & 442747 \\
\hline negative & 310.121 & 18.357 & 7-hydroxy-3-(4-methoxyphenyl)-4-propyl-2H-1-benzopyran-2-one & 5357444 \\
\hline negative & 306.060 & 18.751 & isoprothiolane sulfoxide & 93275 \\
\hline negative & 312.106 & 11.416 & 6-O- $(\beta$-D-xylopyranosyl)- $\beta$-D-glucopyranose & 443248 \\
\hline negative & 814.211 & 3.276 & victorin $\mathrm{C}$ & 21549934 \\
\hline negative & 506.100 & 7.917 & cassiamin $\mathrm{C}$ & 442728 \\
\hline negative & 392.199 & 8.060 & abyssinone VI & 5281219 \\
\hline negative & 610.153 & 1.052 & rutin & 5280805 \\
\hline negative & 372.100 & 0.836 & ohioensin-A & 442531 \\
\hline negative & 594.159 & 10.092 & vitexin $2^{\prime \prime}$-O- $\beta$-D-glucoside & 5280641 \\
\hline negative & 464.096 & 9.183 & quercetin 3-O-glucoside & 5280804 \\
\hline negative & 294.021 & 15.387 & 4-[2,2-dichloro-1-(4-methoxyphenyl)ethenyl]phenol & 156639 \\
\hline negative & 448.101 & 7.367 & isoorientin & 114776 \\
\hline negative & 360.085 & 15.746 & thymonin & 442662 \\
\hline negative & 575.058 & 16.657 & isopentenyladenosine- $5^{\prime}$-triphosphate & 23724748 \\
\hline negative & 522.137 & 7.259 & iridin & 5281777 \\
\hline negative & 342.074 & 17.721 & dihydromethylsterigmatocystin & 5280636 \\
\hline negative & 516.127 & 7.769 & 1,3-dicaffeoylquinic acid & 6474640 \\
\hline negative & 580.312 & 11.523 & hordatine $\mathrm{B}$ & 72193633 \\
\hline
\end{tabular}


Table 1. continued

\begin{tabular}{|c|c|c|c|c|}
\hline ESI & exact mass & mass error (ppm) & matched metabolite from database & PubChem ID \\
\hline negative & 870.218 & 14.415 & iresinin I & 11953907 \\
\hline negative & 714.486 & 11.184 & $\left(2^{\prime} S\right)$-deoxymyxol $2^{\prime}$ - $\alpha$-L-fucoside & 23724611 \\
\hline negative & 487.120 & 5.943 & luciferyl sulfate & 11953812 \\
\hline negative & 486.116 & 6.751 & flavonol 3-O-(6-O-malonyl- $\beta$-D-glucoside) & 11953833 \\
\hline negative & 463.074 & 3.945 & $N^{6}$-(1,2-dicarboxyethyl)-AMP & 447145 \\
\hline negative & 286.048 & 2.426 & aureusidin & 5281220 \\
\hline negative & 136.039 & 17.392 & hypoxanthine & 135398638 \\
\hline negative & 198.039 & 3.888 & 2,4-dinitrophenylhydrazine & 3772977 \\
\hline negative & 719.446 & 19.427 & erythromycin $\mathrm{C}$ & 83933 \\
\hline negative & 432.194 & 12.242 & aspulvinone $\mathrm{H}$ & 54675755 \\
\hline negative & 743.204 & 15.328 & cyanidin $3-O-\left(6^{\prime \prime}\right.$-glucosyl-2"-xylosylgalactoside $)$ & 441671 \\
\hline negative & 181.038 & 19.968 & 2-methyl-3-hydroxy-5-formylpyridine-4-carboxylate & 440898 \\
\hline negative & 374.058 & 7.988 & glucocochlearin & 5281135 \\
\hline negative & 364.095 & 19.154 & justicidin B & 442882 \\
\hline negative & 750.140 & 16.841 & UDP- $N$-acetylmuramoyl-L-alanine & 3037124 \\
\hline negative & 560.081 & 17.449 & dTDP-4-oxo-5-C-methyl-L-rhamnose & 443215 \\
\hline negative & 494.121 & 13.369 & $5^{\prime}$-methoxyhydnocarpin-D & 5281879 \\
\hline negative & 538.111 & 9.840 & lithospermic acid & 6441498 \\
\hline negative & 493.098 & 18.917 & MK826 & 443580 \\
\hline negative & 664.382 & 2.599 & phytolaccoside B & 441939 \\
\hline negative & 344.074 & 11.436 & theogallin & 442988 \\
\hline negative & 374.100 & 17.102 & scullcapflavone II & 124211 \\
\hline negative & 164.069 & 10.386 & $\beta$-D-fucose & 439650 \\
\hline negative & 685.357 & 12.321 & avadharidine & 441710 \\
\hline negative & 162.017 & 18.924 & allicin & 65036 \\
\hline negative & 586.314 & 19.530 & 5-oxoavermectin " $2 \mathrm{~b}$ " aglycone & 11953969 \\
\hline negative & 584.310 & 13.431 & lappaconitine & 5281279 \\
\hline negative & 336.059 & 15.581 & 2,2-bis(4-hydroxyphenyl)hexafluoropropane & 73864 \\
\hline negative & 652.315 & 11.849 & thalidasine & 159795 \\
\hline negative & 608.289 & 19.119 & oxyacanthine & 442333 \\
\hline negative & 380.216 & 5.889 & 4,4-difluoro-17- $\beta$-hydroxyandrost-5-en-3-one propionate & 253787 \\
\hline negative & 568.305 & 5.251 & adouetine $\mathrm{Y}$ & 5281578 \\
\hline negative & 626.148 & 4.216 & quercetin $3-O-\beta$-D-glucosyl-( $1 \rightarrow 2)-\beta$-D-glucoside & 5282166 \\
\hline negative & 660.424 & 8.099 & 12-O-palmitoyl-16-hydroxyphorbol 13-acetate & 334044 \\
\hline negative & 1051.605 & 14.892 & aculeacin A & 14315169 \\
\hline negative & 137.905 & 1.711 & $\mathrm{Ba}^{2+}$ & 104810 \\
\hline positive & 305.939 & 2.050 & 3-iodo-4-hydroxyphenylpyruvate & 440184 \\
\hline positive & 542.121 & 11.040 & isochamaejasmin & 390361 \\
\hline positive & 184.023 & 3.201 & 5-hydroxyisourate & 250388 \\
\hline positive & 543.110 & 11.677 & CMP-3-deoxy-D-manno-octulosonate & 445888 \\
\hline positive & 292.132 & 11.326 & SB 206553 & 5163 \\
\hline positive & 146.069 & 6.737 & D-glutamine & 145815 \\
\hline positive & 115.063 & 14.931 & proline & 145742 \\
\hline positive & 129.043 & 1.863 & 5-oxoproline & 7405 \\
\hline positive & 303.137 & 0.083 & evodiamine & 442088 \\
\hline positive & 117.079 & 9.054 & L-valine & 6287 \\
\hline positive & 324.106 & 4.923 & D-fructofuranose $1,2^{\prime}: 2,3^{\prime}$-dianhydride & 440332 \\
\hline positive & 307.084 & 6.429 & glutathione & 124886 \\
\hline positive & 334.057 & 1.241 & nicotinamide D-ribonucleotide & 14180 \\
\hline positive & 450.116 & 4.305 & neoastilbin & 442437 \\
\hline positive & 305.028 & 13.271 & 2,4-dinitro-1-(3-nitrophenoxy)benzene & 221812 \\
\hline positive & 303.007 & 18.316 & 2-(((3,5-dichlorophenyl)carbamoyl)oxy)-2-methyl-3-butenoic acid & 119359 \\
\hline positive & 900.168 & 18.543 & $N$-methylanthraniloyl-CoA & 24883420 \\
\hline positive & 162.032 & 2.679 & umbelliferone & 5281426 \\
\hline positive & 540.163 & 1.405 & cleistanthin A & 442833 \\
\hline positive & 621.109 & 14.679 & cyanidin $3-O-3^{\prime \prime}, 6^{\prime \prime}$-O-dimalonylglucoside & 23724697 \\
\hline positive & 360.085 & 3.860 & 6-methoxyaromadendrin 3-O-acetate & 442415 \\
\hline positive & 522.110 & 1.595 & cefoselis & 5748845 \\
\hline positive & 162.032 & 2.617 & 3-hydroxycoumarin & 13650 \\
\hline positive & 610.132 & 0.389 & gallocatechin- $(4 \alpha \rightarrow 8)$-epigallocatechin & 442682 \\
\hline positive & 341.054 & 18.994 & aristolochic acid & 2236 \\
\hline
\end{tabular}


Table 1. continued

\begin{tabular}{|c|c|c|c|c|}
\hline ESI & exact mass & mass error $(\mathrm{ppm})$ & matched metabolite from database & PubChem ID \\
\hline positive & 492.090 & 14.101 & carmine & 14950 \\
\hline positive & 249.173 & 4.366 & lophocerine & 442313 \\
\hline positive & 338.045 & 7.327 & UK-47265 & 133777 \\
\hline positive & 606.237 & 17.505 & cancentrine & 5462434 \\
\hline positive & 418.324 & 9.894 & $8^{\prime}$-apo- $\beta$-carotenol & 5280991 \\
\hline positive & 436.334 & 8.296 & 2-phytyl-1,4-naphthoquinone & 56927684 \\
\hline positive & 300.063 & 2.857 & kaempferide & 5281666 \\
\hline positive & 416.147 & 0.577 & 1-acetoxypinoresinol & 442831 \\
\hline positive & 348.169 & 1.303 & enalaprilate & 5462501 \\
\hline positive & 892.534 & 2.918 & zeaxanthin diglucoside & 10533723 \\
\hline positive & 183.977 & 11.963 & 3-phosphonooxypyruvate & 105 \\
\hline positive & 801.531 & 14.884 & $\mathrm{PC}(20: 4(8 Z, 11 Z, 14 Z, 17 Z) / 18: 4(6 Z, 9 Z, 12 Z, 15 Z) / 0: 0)$ & none \\
\hline positive & 803.547 & 9.142 & PC(22:6(4Z,7Z,10Z,13Z,16Z,19Z)/16:1(9Z)/0:0) & none \\
\hline positive & 260.116 & 10.860 & maculosin & 119404 \\
\hline positive & 884.542 & 8.209 & $\operatorname{PI}(20: 4(8 Z, 11 Z, 14 Z, 17 Z) / 18: 1(11 Z))$ & 53480105 \\
\hline positive & 743.547 & 10.424 & $\operatorname{PC}(15: 0 / 18: 2(9 Z, 12 Z) / 0: 0)$ & none \\
\hline positive & 777.531 & 14.567 & PC(22:5(7Z,10Z,13Z,16Z,19Z)/14:1(9Z)/0:0) & none \\
\hline positive & 781.562 & 15.183 & $\operatorname{PC}(20: 3(5 Z, 8 Z, 11 Z) / 16: 1(9 Z) / 0: 0)$ & none \\
\hline positive & 755.547 & 15.502 & $\mathrm{PC}(18: 3(6 \mathrm{Z}, 9 \mathrm{Z}, 12 \mathrm{Z}) / 16: 0 / 0: 0)$ & none \\
\hline positive & 779.547 & 14.305 & $\mathrm{PC}(16: 1(9 Z) / 20: 4(5 Z, 8 Z, 11 Z, 14 Z) / 0: 0)$ & none \\
\hline positive & 757.562 & 13.641 & $\operatorname{PE}(15: 0 / 22: 2(13 Z, 16 Z) / 0: 0)$ & none \\
\hline positive & 753.531 & 4.492 & $\operatorname{PC}(20: 3(5 Z, 8 Z, 11 Z) / 14: 1(9 Z) / 0: 0)$ & none \\
\hline positive & 774.528 & 16.736 & oligomycin $\mathrm{C}$ & 5281901 \\
\hline positive & 596.459 & 0.803 & spirilloxanthin & 5366506 \\
\hline positive & 612.475 & 17.956 & $\operatorname{DG}(14: 1(9 Z) / 22: 5(4 Z, 7 Z, 10 Z, 13 Z, 16 Z) / 0: 0)$ & 53477996 \\
\hline positive & 313.914 & 6.671 & tiron & 9001 \\
\hline positive & 739.515 & 0.965 & $\operatorname{PE}(18: 2(9 Z, 12 Z) / 18: 2(9 Z, 12 Z) / 0: 0)$ & none \\
\hline positive & 741.531 & 5.923 & $\operatorname{PE}(18: 1(11 Z) / 18: 2(9 Z, 12 Z) / 0: 0)$ & none \\
\hline positive & 713.500 & 11.815 & $\operatorname{PE}(20: 3(5 Z, 8 Z, 11 Z) / 14: 0 / 0: 0)$ & none \\
\hline positive & 737.500 & 9.720 & $\operatorname{PE}(14: 1(9 Z) / 22: 4(7 Z, 10 Z, 13 Z, 16 Z) / 0: 0)$ & none \\
\hline positive & 206.006 & 0.849 & 3-oxalomalate & 5459790 \\
\hline positive & 336.140 & 2.553 & steroid $O$-sulfate & 439761 \\
\hline positive & 715.515 & 5.771 & $\operatorname{PE}(20: 2(11 Z, 14 Z) / 14: 0 / 0: 0)$ & none \\
\hline positive & 208.001 & 1.808 & stipitatonate & 54746226 \\
\hline positive & 636.341 & 18.127 & ansatrienin A & 5282069 \\
\hline positive & 329.116 & 5.177 & 2,2'-(1-phenyl-1H-1,2,4-triazole-3,5-diyl)bisphenol & 443276 \\
\hline positive & 716.502 & 18.708 & $1^{\prime}$-hydroxy- $\gamma$-carotene glucoside & 23724600 \\
\hline positive & 328.116 & 5.397 & anisatin & 115121 \\
\hline positive & 332.142 & 3.872 & 1-dehydro-9-fluoro-11-oxotestololactone & 253326 \\
\hline positive & 330.139 & 1.981 & $17 \alpha$-chloroethynylestradiol & 245467 \\
\hline positive & 499.297 & 1.360 & tauroursodeoxycholic acid & 9848818 \\
\hline positive & 155.982 & 15.912 & 2-phosphoglycolate & 529 \\
\hline positive & 477.316 & 0.573 & gentamicin $\mathrm{C} 1$ & 72395 \\
\hline positive & 757.599 & 16.260 & $\mathrm{PE}(20: 1(11 \mathrm{Z}) / \mathrm{dm} 18: 0 / 0: 0)$ & none \\
\hline positive & 171.952 & 6.659 & 4-bromophenol & 7808 \\
\hline positive & 168.972 & 12.949 & 2-aminoethylarsonate & 129501 \\
\hline positive & 168.964 & 10.786 & L-selenocysteine & 6326983 \\
\hline
\end{tabular}

conditions because of defense mechanisms in plants. ${ }^{22}$ For example, flavonoids accumulate in the presence of sucrose as defense mechanisms against osmotic stress in plants. ${ }^{20,21,23}$ In these studies, analysis of gene expression or total flavonoid levels revealed that sucrose induces the upregulation of flavonoid biosynthesis. However, to our knowledge, there is no study on the relationship between flavonoid biosynthesis and sucrose levels through metabolite profiles, especially the individual levels of flavonoids.

In this study, the secondary metabolite profile changes in $M$. officinalis were analyzed in response to different levels of sucrose. To accomplish this, we used ultraperformance liquid chromatography-quadrupole time-of-flight mass spectrometry (UPLC-Q-TOF MS), and the metabolite profiles were statistically analyzed using partial least squares-discriminant analysis (PLS-DA) and hierarchical clustering analysis (HCA). These results can be used in understanding the alteration in metabolisms based on the sucrose level and give clues on the molecular breeding of plants for overproducing high-value metabolites.

\section{RESULTS AND DISCUSSION}

Identification of Secondary Metabolites from $M$. officinalis. To analyze the changes in the profile of secondary 


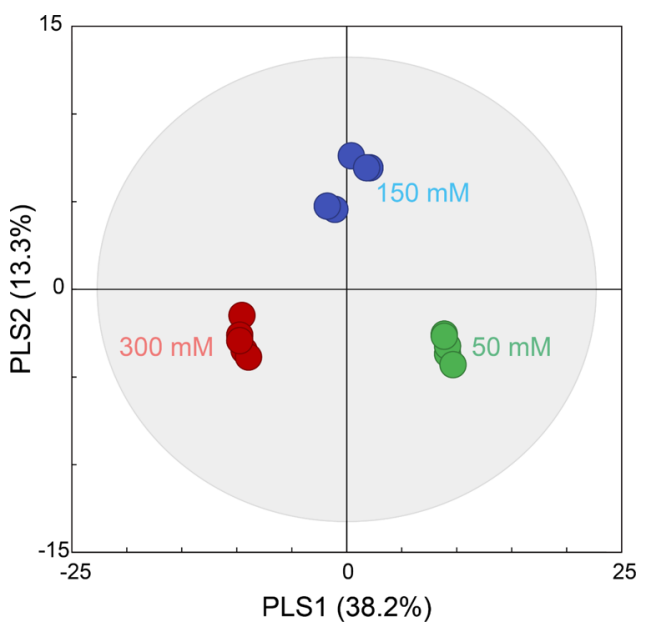

Figure 1. PLS-DA score plot of secondary metabolite profiles in $M$. officinalis treated with 50 (control; green), 150 (blue), and $300 \mathrm{mM}$ (red).

metabolites of $M$. officinalis in response to sucrose, $M$. officinalis leaves treated with 50,150 , or $300 \mathrm{mM}$ sucrose were extracted with $\mathrm{MeOH}$ and analyzed using UPLC-Q-TOF MS. More than 20,000 peaks of the negative electrospray ionization mode $\left(\mathrm{ESI}^{-}\right)$and positive electrospray ionization ions $\left(\mathrm{ESI}^{+}\right)$were detected, and 169 metabolites were identified using XCMS in all the 15 samples obtained from five biological replicates of each condition group (Table 1), indicating that our results were more accurate and less biased than the previous reports that showed metabolic changes under the stressful conditions with 6 unidentified secondary metabolites using LC-MS/ $\mathrm{MS}^{24} 30$ identified secondary metabolites using UPLC-QTOF $\mathrm{MS}^{25}$ and 95 identified metabolites using LC-MS/ MS. $^{26}$ These metabolites were found to be major intermediates in the secondary metabolisms of plants, including the biosynthesis of carotenoids (e.g., (2'S)-deoxymyxol $2^{\prime}-\alpha$-Lfucoside), phenylpropanoids (e.g., 1-O-galloyl- $\beta$-D-glucose, justicidin B, 1-acetoxypinoresinol, 3-hydroxycoumarin, cleistanthin A, and umbelliferone), flavones, and flavonols (e.g., scullcapflavone II, flavonol 3-O-(6-O-malonyl- $\beta$-D-glucoside), cyanidin 3-O-(6"-glucosyl-2"-xylosylgalactoside), iridin, isoorientin, kolaflavanone, thymonin, quercetin 3-O-glucoside, quercetin $3-O-\beta$-D-glucosyl- $(1 \rightarrow 2)-\beta$-D-glucoside, rutin, vitexin 2 "- $O-\beta$-D-glucoside, malvidin, and petunidin).

The secondary metabolites identified in this study are well known to have beneficial health effects. For example, rutin, lithospermic acid, moxalactam, isoorientin, 5'-methoxyhydnocarpin-D, oxyacanthine, 1,3-dicaffeoylquinic acid, isohelenol, lappaconitine, phytolaccoside B, iridin, and scullcapflavone II are known to possess various physiological activities such as antioxidative, ${ }^{27}$ antibacterial, ${ }^{28}$ hepatoprotective, ${ }^{29}$ anti-HIV$1,^{30}$ antifungal, $^{31}$ antimutagenic, ${ }^{32}$ and anti-inflammatory ${ }^{33}$ activities. Specifically, malvidin, a primary plant pigment, inhibits human leukemia cells by arresting the $\mathrm{G}_{2} / \mathrm{M}$ phase and then inducing apoptosis. ${ }^{34}$ Lithospermic acid can be used in diabetic retinopathy and mesenteric ischemia reperfusion injury because of its antioxidative, hepatoprotective, and antiinflammatory effects. ${ }^{27,35}$

PLS-DA of the Sucrose Effect on Secondary Metabolite Profiles. To statistically compare changes in the profile of secondary metabolites of $M$. officinalis in response to different levels of sucrose, principal component analysis (PCA) was performed using SIMCA-P+. Because the metabolite profiles of the groups were slightly discriminated by PCA, with 0.52 of $R^{2} X$ and 0.33 of $Q^{2}$ (data not shown), PLS-DA was employed to obtain better separations between the groups. Among the three groups treated with sucrose levels of 50, 150, and $300 \mathrm{mM}$, the metabolite profiles were clearly separated by partial least squares 1 (PLS1) and 2 (PLS2) in the score plot of PLS-DA (Figure 1). The model generated explained variation values, such as 0.52 of $R^{2} X$ and 0.97 of $R^{2} Y$, and a predictive capability value, such as 0.87 of cumulative $Q^{2}$, indicating a good model. Our previous study on the change in flavonoid levels in lemon balm by sucrose also showed that six

Table 2. Top 20 Identified Metabolites with High Absolute Loadings on PLS1 and PLS2 as Determined by PLS-DA

\begin{tabular}{|c|c|c|c|}
\hline \multicolumn{2}{|l|}{ PLS1 } & \multicolumn{2}{|l|}{ PLS2 } \\
\hline metabolite & loading & metabolite & loading \\
\hline anisatin & 0.113 & 10-formyldihydrofolate & 0.197 \\
\hline 2,2'-(1-phenyl-1H-1,2,4-triazole-3,5-diyl)bisphenol & 0.107 & 2,4-dinitrophenylhydrazine & 0.159 \\
\hline quercetin $3-O-\beta$-D-glucosyl- $(1 \rightarrow 2)-\beta$-D-glucoside & 0.107 & 2-carboxy-D-arabinitol 1-phosphate & 0.157 \\
\hline isohelenol & 0.101 & 2-methyl-3-hydroxy-5-formylpyridine-4-carboxylate & 0.112 \\
\hline L-aspartate & 0.092 & $5^{\prime}$-methoxyhydnocarpin-D & 0.138 \\
\hline thalidasine & 0.091 & 7-hydroxy-6-methyl-8-ribityl lumazine & 0.134 \\
\hline aspulvinone $\mathrm{H}$ & 0.091 & allicin & 0.132 \\
\hline 2,2-bis(4-hydroxyphenyl)hexafluoropropane & 0.091 & $\alpha$-oxo-benzeneacetic acid & 0.130 \\
\hline allicin & 0.090 & $\mathrm{Ba}^{2+}$ & 0.120 \\
\hline 6-O-( $\beta$-D-xylopyranosyl)- $\beta$-D-glucopyranose & 0.090 & butanoylphosphate & 0.115 \\
\hline isopentenyladenosine- $5^{\prime}$-triphosphate & -0.115 & CMP-3-deoxy-D-manno-octulosonate & -0.107 \\
\hline 6-methoxyaromadendrin 3-O-acetate & -0.115 & DG(14:1(9Z)/22:5(4Z,7Z,10Z,13Z,16Z)/0:0) & -0.107 \\
\hline BMS-268770 & -0.115 & 5-oxoproline & -0.109 \\
\hline 2,4-dinitrophenylhydrazine & -0.115 & 1-dehydro-9-fluoro-11-oxotestololactone & -0.115 \\
\hline gentamicin $\mathrm{C} 1$ & -0.115 & cassiamin $\mathrm{C}$ & -0.121 \\
\hline iridin & -0.118 & cellotriose & -0.121 \\
\hline 3-hydroxycoumarin & -0.118 & victorin $\mathrm{C}$ & -0.130 \\
\hline cefoselis & -0.119 & rutin & -0.132 \\
\hline lithospermic acid & -0.119 & thalidasine & -0.132 \\
\hline dihydromethylsterigmatocystin & -0.123 & quercetin $3-O-\beta$-D-glucosyl- $(1 \rightarrow 2)-\beta$-D-glucoside & -0.141 \\
\hline
\end{tabular}


Table 3. VIP Scores of the 78 Metabolites with a VIP >1.0 That Strongly Contributed to the PLS-DA Model

\begin{tabular}{|c|c|}
\hline metabolite & VIP \\
\hline 2-methyl-3-hydroxy-5-formylpyridine-4-carboxylate & 1.772 \\
\hline narciclasine & 1.598 \\
\hline glutathione & 1.588 \\
\hline allicin & 1.500 \\
\hline$\alpha$-oxo-benzeneacetic acid & 1.417 \\
\hline quercetin $3-O-\beta$-D-glucosyl-( $1 \rightarrow 2)-\beta$-D-glucoside & 1.400 \\
\hline $\operatorname{PE}(15: 0 / 22: 2(13 Z, 16 Z) / 0: 0)$ & 1.400 \\
\hline phytolaccoside B & 1.378 \\
\hline$N^{6}$-(1,2-dicarboxyethyl)-AMP & 1.377 \\
\hline iresinin I & 1.375 \\
\hline rutin & 1.366 \\
\hline 10-formyldihydrofolate & 1.363 \\
\hline umbelliferone & 1.360 \\
\hline cassiamin $\mathrm{C}$ & 1.359 \\
\hline $\operatorname{PC}(18: 3(6 Z, 9 Z, 12 Z) / 16: 0 / 0: 0)$ & 1.355 \\
\hline victorin $\mathrm{C}$ & 1.350 \\
\hline 2-carboxy-D-arabinitol 1-phosphate & 1.343 \\
\hline cleistanthin A & 1.338 \\
\hline 2,4-dinitrophenylhydrazine & 1.332 \\
\hline 5'-methoxyhydnocarpin-D & 1.330 \\
\hline gentamicin $\mathrm{C} 1$ & 1.318 \\
\hline 6-methoxyaromadendrin 3-O-acetate & 1.317 \\
\hline flavonol 3-O-(6-O-malonyl- $\beta$-D-glucoside) & 1.316 \\
\hline luciferyl sulfate & 1.311 \\
\hline erythromycin C & 1.302 \\
\hline 5-oxoproline & 1.297 \\
\hline 1-dehydro-9-fluoro-11-oxotestololactone & 1.290 \\
\hline 3-hydroxycoumarin & 1.279 \\
\hline proline & 1.274 \\
\hline thymonin & 1.271 \\
\hline L-selenocysteine & 1.252 \\
\hline cefoselis & 1.250 \\
\hline PC(22:5(7Z,10Z,13Z,16Z,19Z)/14:1(9Z)/0:0) & 1.233 \\
\hline thalidasine & 1.226 \\
\hline L-aspartate & 1.214 \\
\hline 7-hydroxy-6-methyl-8-ribityl lumazine & 1.211 \\
\hline aspulvinone $\mathrm{H}$ & 1.198 \\
\hline 2,2-bis(4-hydroxyphenyl)hexafluoropropane & 1.197 \\
\hline $\mathrm{Ba}^{2+}$ & 1.192 \\
\hline
\end{tabular}

\begin{tabular}{|c|c|}
\hline metabolite & VIP \\
\hline catechol & 1.190 \\
\hline scullcapflavone II & 1.182 \\
\hline lithospermic acid & 1.173 \\
\hline$N$-methylanthraniloyl-CoA & 1.170 \\
\hline iridin & 1.168 \\
\hline gallocatechin- $(4 \alpha \rightarrow 8)$-epigallocatechin & 1.168 \\
\hline 2-(((3,5-dichlorophenyl)carbamoyl)oxy)-2-methyl-3-butenoic acid & 1.158 \\
\hline PC(22:6(4Z,7Z,10Z,13Z,16Z,19Z)/16:1(9Z)/0:0) & 1.156 \\
\hline dihydromethylsterigmatocystin & 1.151 \\
\hline vitexin $2^{\prime \prime}-O-\beta$-D-glucoside & 1.149 \\
\hline evodiamine & 1.143 \\
\hline lophocerine & 1.142 \\
\hline CMP-3-deoxy-D-manno-octulosonate & 1.138 \\
\hline aureusidin & 1.116 \\
\hline INF271 & 1.115 \\
\hline BQ 518 & 1.113 \\
\hline tauroursodeoxycholic acid & 1.110 \\
\hline isohelenol & 1.104 \\
\hline hypoxanthine & 1.101 \\
\hline isopentenyladenosine- 5 '-triphosphate & 1.093 \\
\hline 2,2'-(1-phenyl-1H-1,2,4-triazole-3,5-diyl)bisphenol & 1.082 \\
\hline $\operatorname{PC}(20: 3(5 Z, 8 Z, 11 Z) / 16: 1(9 Z) / 0: 0)$ & 1.075 \\
\hline UDP-N-acetylmuramoyl-L-alanine & 1.065 \\
\hline $\operatorname{PE}(20: 3(5 Z, 8 Z, 11 Z) / 14: 0 / 0: 0)$ & 1.060 \\
\hline cellotriose & 1.057 \\
\hline BMS-268770 & 1.056 \\
\hline 3-iodo-4-hydroxyphenylpyruvate & 1.056 \\
\hline 6-(isopropylthio)purine & 1.053 \\
\hline carmine & 1.053 \\
\hline butanoylphosphate & 1.046 \\
\hline DG(14:1(9Z)/22:5(4Z,7Z,10Z,13Z,16Z)/0:0) & 1.045 \\
\hline anisatin & 1.043 \\
\hline 4-[2,2-dichloro-1-(4-methoxyphenyl)ethenyl]phenol & 1.042 \\
\hline $\mathrm{PI}(20: 4(8 Z, 11 Z, 14 Z, 17 Z) / 18: 1(11 Z))$ & 1.040 \\
\hline isoorientin & 1.034 \\
\hline quercetin 3-O-glucoside & 1.017 \\
\hline cefaloglycin & 1.015 \\
\hline avadharidine & 1.013 \\
\hline justicidin B & 1.009 \\
\hline
\end{tabular}

metabolite profiles were significantly different between 50,150 , and $300 \mathrm{mM}$ sucrose. ${ }^{24}$ However, the present results may be considered more accurate and reliable because only six secondary metabolites (i.e., 435.13, 523.129, 540.063, $573.200,615.714$, and 617.153) were used in the previous study without identification. In the permutation test, all points of permuted $R^{2}$ and $Q^{2}$ values to the left were located in the lower side contrary to the original points, and the regression line of $Q^{2}$ had a negative intercept, indicating that the PLS-DA models were clearly validated without overfitting from the original model (Figure S2). ${ }^{36}$

The loading scores of the selected 20 metabolites, which represented the magnitude of the contribution of each metabolite to PLS, are listed in Table 2. Of the identified 169 metabolites in this study, 40 metabolites including anisatin, quercetin $3-O-\beta$-D-glucosyl- $(1 \rightarrow 2)-\beta$-D-glucoside, isohelenol, and L-aspartate contributed positively to PLS1. However, 129 metabolites such as lithospermic acid, iridin, 3-hydroxycoumarin, and 6-methoxyaromadendrin 3-O-acetate contributed negatively to PLS1. Seventy-nine metabolites including 10-formyldihydrofolate, 2,4-dinitrophenylhydrazine, and allicin contributed positively to PLS2, while 90 metabolites such as quercetin 3-O- $\beta$-D-glucosyl- $(1 \rightarrow 2)-\beta$-D-glucoside, rutin, thalidasine, and victorin $\mathrm{C}$ contributed negatively to PLS2.

In variable importance in projection (VIP) analysis, VIP values greater than 1 are considered important. ${ }^{36}$ In this study, 78 metabolites such as quercetin $3-O-\beta$-D-glucosyl- $(1 \rightarrow 2)-\beta$-Dglucoside, rutin, umbelliferone, and cleistanthin A were shown to have VIP values greater than 1, of which 16 metabolites belong to flavonoid classes (Table 3 ). These results suggested that the flavonoids were critical metabolites for discriminating between the groups.

HCA of the Sucrose Effect on Secondary Metabolite Profiles. To cluster and visualize the discrimination of secondary metabolite profiles with 50, 150, and $300 \mathrm{mM}$ sucrose, HCA with the Euclidean distance coefficient and average linkage was performed using $\mathrm{MeV}$ software. After normalization using the sum of identified metabolites and then transformation using unit variance scaling, data composed of 


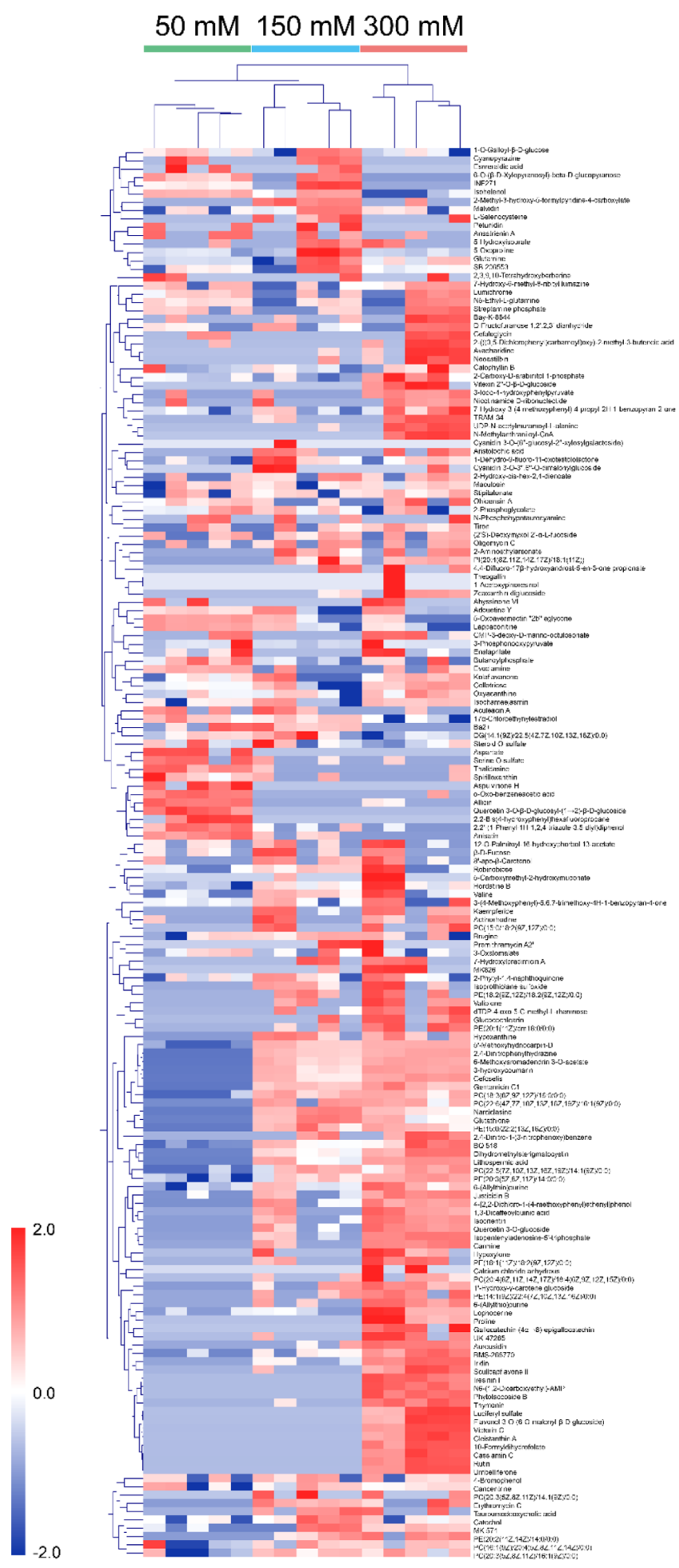

Figure 2. Clustered heat map of 169 secondary metabolites of $M$. officinalis treated with 50 (control; green), 150 (blue), and $300 \mathrm{mM}$ (red) sucrose. Similarity assessment of clustering based on the Euclidean distance coefficient and average linkage method. Each column and each row represent different concentrations of sucrose and individual metabolite, respectively.

identified metabolites and groups (50, 150, and $300 \mathrm{mM}$ sucrose) were exported into the heat map.

In the heat map, five biological replicates at each group had similar metabolite profiles (Figure 2). However, the metabolite profiles were significantly different depending on different sucrose levels, 50, 150, and $300 \mathrm{mM}$. The secondary metabolite profile of $150 \mathrm{mM}$ sucrose was closer to that of $300 \mathrm{mM}$ sucrose than to that of $50 \mathrm{mM}$ sucrose. These results are similar to those obtained in a previous study on primary metabolite profiles in M. officinalis with 64 metabolites. ${ }^{4}$ This comparison indicates that the effect of sucrose level on primary metabolite profiles may be associated with the secondary metabolite profiles in M. officinalis. Moreover, the clustering of secondary metabolite profiles between sucrose levels was enabled by certain individual metabolites. For example, Lserine $O$-sulfate, thalidasin, spirilloxanthin, and quercetin 3-O$\beta$-D-glucosyl- $(1 \rightarrow 2)$ - $\beta$-D-glucoside increased in $50 \mathrm{mM}$. However, the levels of proline, glutathione, isoorientin scullcapflavone II, flavonol 3-O-(6-O-malonyl- $\beta$-D-glucoside), luciferyl sulfate, cassiamin $\mathrm{C}$, and rutin were much higher in $300 \mathrm{mM}$ sucrose than in 50 and $150 \mathrm{mM}$ sucrose.

Comparison of Individual Flavonoid Levels with 50, 150, and $300 \mathrm{mM}$ Sucrose. Most studies have reported only total flavonoid abundances to reveal the relationship between sucrose levels and contents of total flavonoids ${ }^{20,24,37,38}$ or the phenylpropanoid pathway ${ }^{39,40}$ without identifying or comparing individual flavonoid abundances. In this study, we identified individual secondary metabolites and determined the changes in each flavonoid, anthocyanindin, and phenlypropanoid levels depending on sucrose levels.

To compare the changes in flavonoid level between the groups, one-way analysis of variance with the post hoc Tukey's honestly significant difference test was conducted using Statistica $(p>0.05)$. The abundance of three flavonoids such as quercetin 3- $O-\beta$-D-glucosyl- $(1 \rightarrow 2)-\beta$-D-glucoside, 6 methoxyaromadendrin 3-O-acetate, and 3-hydroxycoumarin increased with $150 \mathrm{mM}$ sucrose compared to those with 50 $\mathrm{mM}$ sucrose. However, compared to those with $50 \mathrm{mM}$ sucrose, the abundances of most flavonoids such as 6methoxyaromadendrin 3-O-acetate, 3-hydroxycoumarin, aureusidin, thymonin, rutin, justicidin B, isoorientin, quercetin 3$O$-glucoside, umbelliferone, iridin, scullcapflavone II, cleistanthin A, flavonol 3-O-(6-O-malonyl- $\beta$-glucoside), isochamaejasmin, gallocatechin- $(\alpha \rightarrow 8)$-epigallocatechin, vitexin $2^{\prime \prime}-O-\beta$ glucoside, kolaflavanone, kaempferide, and neoastilbin were significantly increased with $300 \mathrm{mM}$ (Figure 3). These results showed that flavonoids accumulated depending on the sucrose level, indicating that sucrose induced the production of more flavonoids via the phenylpropanoid pathway.

Similar to these results, previous studies have reported that rutin accumulates in Fagopyrum esculentum Moench in response to sucrose $\mathrm{e}^{41}$ and quercetin 3-O-glucoside accumulates in Arabidopsis under abiotic and oxidative stress. ${ }^{42}$ Our results showed that the types of accumulating flavonoids in $M$. officinalis differed depending on sucrose levels, and active flavonoid biosynthesis served as a defense mechanism against osmotic stress, suggesting that the biosynthetic pathway of flavonoids was regulated by the sucrose signaling pathway. Previously, it was observed at the messenger RNA level that sucrose caused the accumulation of anthocyanins and the upregulation of anthocyanin synthesis. ${ }^{20}$ However, our results showed that anthocyanins (e.g., malvidin and petunidin) did not accumulate under a high sucrose level at both 150 and 300 $\mathrm{mM}$. This is possibly because anthocyanins other than malvidin and petunidin were not identified in this study, and malvidin and petunidin could not represent the behaviors of all other anthocyanins under a high sucrose level. The precise prediction and speculation of the secondary metabolism and 


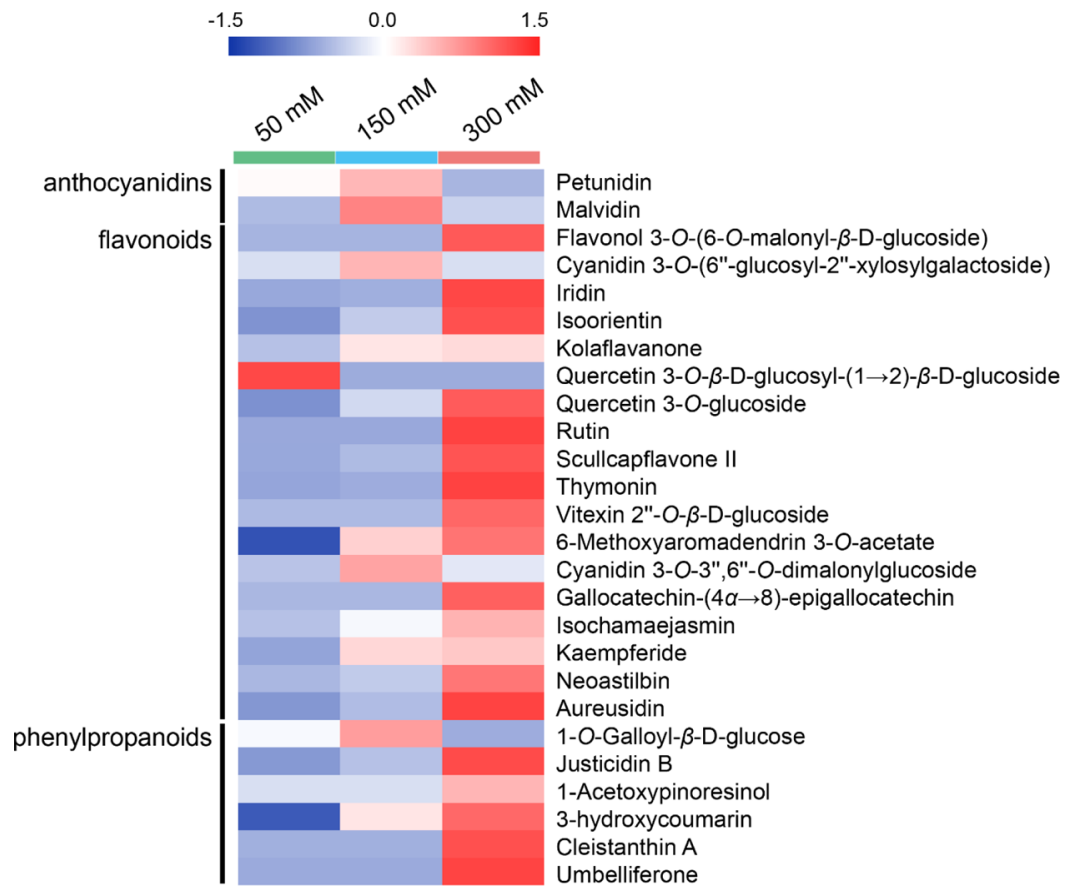

Figure 3. Heat map of 26 flavonoids in M. officinalis treated with 50 (control; green), 150 (blue), and $300 \mathrm{mM}$ (red). Each row represents individual flavonoids.

change in individual secondary metabolites of M. officinalis in response to the concentrations of sucrose should be supported and verified by further experiments.

\section{CONCLUSIONS}

This is the first report to investigate the change in secondary metabolite profiles in $M$. officinalis depending on the sucrose level using UPLC-Q-TOF MS. One hundred and sixty-nine metabolites were identified using XCMS; these metabolites were major intermediates in the secondary metabolism of plants such as the biosynthesis of carotenoids, phenylpropanoids, flavones, and flavonols, which serves as a defense mechanism against stress in plants. PLS-DA and HCA results showed a significant difference in secondary metabolite profiles in $M$. officinalis between 50, 150, and $300 \mathrm{mM}$ sucrose. In contrast to that with $50 \mathrm{mM}$ sucrose, 32 secondary metabolites such as 6-methoxyaromadendrin 3-O-acetate and 3-hydroxycoumarin accumulated in $150 \mathrm{mM}$, and 76 metabolites such as aureusidin, thymonin, quercetin 3-O-glucoside, and rutin increased in $300 \mathrm{mM}$. Accumulation of different types of flavonoids was observed depending on the sucrose level, suggesting that the accumulation of these flavonoids acts as a defense mechanism against osmotic stress. This study demonstrated that secondary metabolite profiles could be a useful tool for investigating the change in certain secondary metabolites and secondary metabolism in plants under osmotic stress and provide clues for manipulating plant metabolisms to produce target flavonoids, which have various properties such as antitumoral, antioxidant, antifungal, and antibacterial activities.

\section{MATERIALS AND METHODS}

Plant Growth Conditions. M. officinalis was prepared as previously described. ${ }^{4}$ Briefly, $M$. officinalis was cultivated in 4 $\mathrm{g} / \mathrm{L}$ Murashige and Skoog medium $\left(0.025 \mathrm{mg} / \mathrm{L}\right.$ of $\mathrm{CoCl}_{2}$. $6 \mathrm{H}_{2} \mathrm{O}, 0.025 \mathrm{mg} / \mathrm{L}$ of $\mathrm{CuSO}_{4} \cdot 5 \mathrm{H}_{2} \mathrm{O}, 36.70 \mathrm{mg} / \mathrm{L}$ of
FeNaEDTA, $6.20 \mathrm{mg} / \mathrm{L}$ of $\mathrm{H}_{3} \mathrm{BO}_{3}, 0.83 \mathrm{mg} / \mathrm{L}$ of $\mathrm{KI}, 16.9$ $\mathrm{mg} / \mathrm{L}$ of $\mathrm{MnSO}_{4} \cdot \mathrm{H}_{2} \mathrm{O}, 0.25 \mathrm{mg} / \mathrm{L}$ of $\mathrm{Na}_{2} \mathrm{MoO}_{4} \cdot 2 \mathrm{H}_{2} \mathrm{O}, 8.60$ $\mathrm{mg} / \mathrm{L}$ of $\mathrm{ZnSO}_{4} \cdot 7 \mathrm{H}_{2} \mathrm{O}, 332.02 \mathrm{mg} / \mathrm{L}$ of $\mathrm{CaCl}_{2}, 170.00 \mathrm{mg} / \mathrm{L}$ of $\mathrm{KH}_{2} \mathrm{PO}_{4}, 1900.00 \mathrm{mg} / \mathrm{L}$ of $\mathrm{KNO}_{3}, 180.54 \mathrm{mg} / \mathrm{L}$ of $\mathrm{MgSO}_{4}$, and $1650.00 \mathrm{mg} / \mathrm{L}$ of $\mathrm{NH}_{4} \mathrm{NO}_{3}$ ) containing $50 \mathrm{mM}$ sucrose and $7 \mathrm{~g} / \mathrm{L}$ agar at $\mathrm{pH} 5.7$ after $2 \mathrm{~cm}$-long explants with two leaves were transferred to the culture and test media with three different concentrations of sucrose, 50 (control), 150, or 300 $\mathrm{mM}$, for examining the effects of sucrose concentration on flavonoid accumulation in M. officinalis. ${ }^{4,24}$ The leaves were incubated at $25^{\circ} \mathrm{C}$ for 20 days (15:9 h light-dark cycle). The leaves of $M$. officinalis were harvested and quickly frozen in liquid nitrogen to quench cellular metabolism, and the frozen samples were stored at $-80{ }^{\circ} \mathrm{C}$.

Metabolite Extraction and UPLC-Q-TOF MS Analysis. Fifty milligrams of ground $M$. officinalis leaves were extracted with $0.5 \mathrm{~mL}$ of cold methanol (high-performance liquid chromatography grade, Merck, Darmstadt, Germany). The methanol extract was diluted with $50 \mu \mathrm{L}$ and was thoroughly vortexed, after which it was centrifuged at $14,000 \mathrm{~g}$ for $5 \mathrm{~min}$. The supernatant was filtered using a $0.45 \mu \mathrm{m}$ syringe filter (hydrophilic poly(tetrafluoroethylene), Advantec, Dublin, $\mathrm{OH})$. The metabolite extract was stored at $-20{ }^{\circ} \mathrm{C}$ before UPLC-Q-TOF MS analysis.

Metabolite extract was analyzed by UPLC-Q-TOF MS. The UPLC analysis was performed using a Waters ACQUITY UPLC system (Waters, Milford, MA) equipped with a Waters ACQUITY BEH C18 column $(100 \times 2.1 \mathrm{~mm}, 1.7 \mu \mathrm{m})$. The mobile phase consisted of solvent $\mathrm{A}, 0.1 \%(\mathrm{w} / \mathrm{v})$ formic acid in distilled water, and solvent $\mathrm{B}, 0.1 \%(\mathrm{w} / \mathrm{v})$ formic acid in acetonitrile. The UPLC was eluted first with a linear gradient from 10 to $100 \%$ of solvent $B(0-7.0 \mathrm{~min})$ and then eluted isocratically with $100 \%$ of solvent $\mathrm{B}(7.0-8.0 \mathrm{~min})$. The flow rate was $0.3 \mathrm{~mL} / \mathrm{min}$, and the injection volume was $5 \mu \mathrm{L}$. The column and autosampler were maintained at 35 and $15{ }^{\circ} \mathrm{C}$, respectively. Mass spectrometry was performed using a Q-TOF 
micromass detector (Waters, Manchester, UK). The conditions of the Q-TOF mass spectrometer in the negative electrospray ionization (ESI) mode were $2800 \mathrm{~V}$ of capillary voltage, $35 \mathrm{~V}$ of sample cone voltage, $1.0 \mathrm{~V}$ of extraction cone voltage, $250{ }^{\circ} \mathrm{C}$ of desolvation temperature, $100{ }^{\circ} \mathrm{C}$ of source temperature, and $500 \mathrm{~L} / \mathrm{h}$ of desolvation gas flow rate. The positive ESI was under the same conditions, expect for an extraction cone voltage of $2.0 \mathrm{~V}$. The ESI mass spectra were acquired over $m / z 100-1500$. Leucine-enkephalin was used as a reference ion by the LockSpray interface to measure mass more accurately and reproducibly.

Data Processing and Statistical Analysis. Acquired data were analyzed using Waters MassLynx (version 4.1). The noise elimination level was set at 6.0 with 10 masses per retention time being collected. Before further processing, lock spray scans were removed because lock spray peaks disrupted the detection and analysis of actual signals from samples (Figure S1A,B). UPLC-Q-TOF MS data were preprocessed using $\mathrm{XCMS}$ with signal-to-noise ratios as described in the literature (Table S1). ${ }^{43,44}$ Mass and retention time windows were set at $0.05 \mathrm{Da}$ and $0.20 \mathrm{~min}$, respectively. After normalization by $\log$ transformation, the processed data were further analyzed using PLS-DA and HCA with the Euclidean distance coefficient and average linkage methods. SIMCA-P+ (version 14.1, Umetrics $\mathrm{AB}$, Umea, Sweden) was used for PLS-DA, ${ }^{36}$ and $\mathrm{MeV}$ (MultiExperiment Viewer; Dana-Farber Cancer Institute, Boston, MA) was used for $\mathrm{HCA}^{45}$ Statistica (version 7.1; StatSoft, Tulsa, OK) was used for the univariate analysis. ${ }^{46}$

\section{ASSOCIATED CONTENT}

\section{SI Supporting Information}

The Supporting Information is available free of charge at https://pubs.acs.org/doi/10.1021/acsomega.0c04745.

Chromatograms for the same samples before and after removal of lock spray scans as examples; validation of the PLS-DA model using the 100 permutation test; and number of peaks detected, peak groups, IP clusters, and predictions in negative and positive modes (PDF)

\section{AUTHOR INFORMATION}

\section{Corresponding Authors}

Dong-Yup Lee - School of Chemical Engineering, Sungkyunkwan University, Suwon 25308, South Korea; Email: dongyuplee@skku.edu

Kyoung Heon Kim - Department of Biotechnology, Graduate School, Korea University, Seoul 02841, South Korea; (1) orcid.org/0000-0003-4600-8668; Phone: +82-2-32903028; Email: khekim@korea.ac.kr

\section{Authors}

Sooah Kim - Department of Environment Science and Biotechnology, Jeonju University, Jeonju 55069, South Korea

Jungyeon Kim - Department of Biotechnology, Graduate School, Korea University, Seoul 02841, South Korea

Nahyun Kim - College of Life Sciences and Biotechnology, Korea University, Seoul 02841, South Korea

Dongho Lee - College of Life Sciences and Biotechnology, Korea University, Seoul 02841, South Korea; 이이.org/ 0000-0003-4379-814X

Hojoung Lee - College of Life Sciences and Biotechnology, Korea University, Seoul 02841, South Korea; $\odot$ orcid.org/ 0000-0002-5626-5695
Complete contact information is available at:

https://pubs.acs.org/10.1021/acsomega.0c04745

\section{Author Contributions}

K.H.K. and D.L. conceived and designed the project. S.K. and H.L. collected the samples. S.K., J.K., and N.K. performed the experiments. S.K., J.K., N.K., D.-Y.L., and D.L. acquired the metabolomics data. S.K., D.L., H.L., D.-Y.L., and K.H.K. analyzed the data. S.K., D.-Y.L., and K.H.K. wrote the manuscript.

Notes

The authors declare no competing financial interest.

\section{ACKNOWLEDGMENTS}

This work was supported by the Mid-career Researcher Program and Young Researcher Program from the National Research Foundation of Korea (NRF-2020R1A2B5B02002631 and NRF-2020R1G1A100826811, respectively). S.K. acknowledges the support of the Research Grant of Jeonju University in 2019. Facility support at the Korea University Food Safety Hall by the Institute of Biomedical Science and Food Safety is also acknowledged.

\section{REFERENCES}

(1) Fiehn, O. Metabolomics-the link between genotypes and phenotypes. Plant Mol. Biol. 2002, 48, 155-171.

(2) Weckwerth, W. Metabolomics in systems biology. Annu. Rev. Plant Biol. 2003, 54, 669-689.

(3) Fiehn, O.; Kopka, J.; Trethewey, R. N.; Willmitzer, L. Identification of uncommon plant metabolites based on calculation of elemental compositions using gas chromatography and quadrupole mass spectrometry. Anal. Chem. 2000, 72, 3573-3580.

(4) Kim, S.; Shin, M. H.; Hossain, M. A.; Yun, E. J.; Lee, H.; Kim, K. $\mathrm{H}$. Metabolite profiling of sucrose effect on the metabolism of Melissa officinalis by gas chromatography-mass spectrometry. Anal. Bioanal. Chem. 2011, 399, 3519-3528.

(5) Theodoridis, G.; Gika, H.; Franceschi, P.; Caputi, L.; Arapitsas, P.; Scholz, M.; Masuero, D.; Wehrens, R.; Vrhovsek, U.; Mattivi, F. LC-MS based global metabolite profiling of grapes: solvent extraction protocol optimisation. Metabolomics 2012, 8, 175-185.

(6) Matsuda, F.; Yonekura-Sakakibara, K.; Niida, R.; Kuromori, T.; Shinozaki, K.; Saito, K. MS/MS spectral tag-based annotation of nontargeted profile of plant secondary metabolites. Plant J. 2009, 57, $555-577$

(7) Krishnan, P.; Kruger, N. J.; Ratcliffe, R. G. Metabolite fingerprinting and profiling in plants using NMR. J. Exp. Bot. 2005, $56,255-265$.

(8) Bundy, J. G.; Spurgeon, D. J.; Svendsen, C.; Hankard, P. K.; Osborn, D.; Lindon, J. C.; Nicholson, J. K. Earthworm species of the genus Eisenia can be phenotypically differentiated by metabolic profiling. FEBS Lett. 2002, 521, 115-120.

(9) Fraser, P. D.; Pinto, M. E. S.; Holloway, D. E.; Bramley, P. M. Application of high-performance liquid chromatography with photodiode array detection to the metabolic profiling of plant isoprenoids. Plant J. 2000, 24, 551-558.

(10) Sumner, L. W.; Mendes, P.; Dixon, R. A. Plant metabolomics: large-scale phytochemistry in the functional genomics era. Phytochemistry 2003, 62, 817-836.

(11) Tagashira, M.; Ohtake, Y. A new antioxidative 1,3-benzodioxole from Melissa officinalis. Planta Med. 1998, 64, 555-558.

(12) de Sousa, A. C.; Gattass, C. R.; Alviano, D. S.; Alviano, C. S.; Blank, A. F.; Alves, P. B. Melissa officinalis L. essential oil: antitumoral and antioxidant activities. J. Pharm. Pharmacol. 2004, 56, 677-681.

(13) Kim, S.; Yun, E. J.; Bak, J. S.; Lee, H.; Lee, S. J.; Kim, C. T.; Lee, J.-H.; Kim, K. H. Response surface optimised extraction and 
chromatographic purification of rosmarinic acid from Melissa officinalis leaves. Food Chem. 2010, 121, 521-526.

(14) Nikitina, V. S.; Kuz'mina, L. Y.; Melent'ev, A. I.; Shendel', G. V. Antibacterial activity of polyphenolic compounds isolated from plants of geraniaceae and rosaceae families. Appl. Biochem. Microbiol. 2007, 43, 629-634.

(15) Viollon, C.; Chaumont, J.-P. Antifungal properties of essential oils and their main components upon Cryptococcus neoformans. Mycopathologia 1994, 128, 151-153.

(16) Miron, T. L.; Herrero, M.; Ibáñez, E. Enrichment of antioxidant compounds from lemon balm (Melissa officinalis) by pressurized liquid extraction and enzyme-assisted extraction. J. Chromatogr. A 2013, 1288, 1-9.

(17) Weitzel, C.; Petersen, M. Enzymes of phenylpropanoid metabolism in the important medicinal plant Melissa officinalis L. Planta 2010, 232, 731-742.

(18) Kong, J.-Q. Phenylalanine ammonia-lyase, a key component used for phenylpropanoids production by metabolic engineering. RSC Adv. 2015, 5, 62587-62603.

(19) Smeekens, S.; Hellmann, H. A. Sugar sensing and signaling in plants. Front. Plant Sci. 2014, 5, 113.

(20) Solfanelli, C.; Poggi, A.; Loreti, E.; Alpi, A.; Perata, P. Sucrosespecific induction of the anthocyanin biosynthetic pathway in Arabidopsis. Plant Physiol. 2006, 140, 637-646.

(21) Zheng, Y.; Tian, L.; Liu, H.; Pan, Q.; Zhan, J.; Huang, W. Sugars induce anthocyanin accumulation and flavanone 3-hydroxylase expression in grape berries. Plant Growth Regul. 2009, 58, 251-260.

(22) Bennett, R. N.; Wallsgrove, R. M. Secondary metabolites in plant defence mechanisms. New Phytol. 1994, 127, 617-633.

(23) Lloyd, J. C.; Zakhleniuk, O. V. Responses of primary and secondary metabolism to sugar accumulation revealed by microarray expression analysis of the Arabidopsis mutant, pho3. J. Exp. Bot. 2004, $55,1221-1230$.

(24) Hossain, M. A.; Kim, S.; Kim, K. H.; Lee, S.-J.; Lee, H. Flavonoid compounds are enriched in lemon balm (Melissa officinalis) leaves by a high level of sucrose and confer increased antioxidant activity. Hortscience 2009, 44, 1907-1913.

(25) Pant, B.-D.; Pant, P.; Erban, A.; Huhman, D.; Kopka, J.; Scheible, W.-R. Identification of primary and secondary metabolites with phosphorus status-dependent abundance in Arabidopsis, and of the transcription factor PHR1 as a major regulator of metabolic changes during phosphorus limitation. Plant, Cell Environ. 2015, 38, $172-187$.

(26) Matsuda, F.; Yonekura-Sakakibara, K.; Niida, R.; Kuromori, T.; Shinozaki, K.; Saito, K. MS/MS spectral tag-based annotation of nontargeted profile of plant secondary metabolites. Plant J. 2009, 57, 555-577.

(27) Jin, C. J.; Yu, S. H.; Wang, X.-M.; Woo, S. J.; Park, H. J.; Lee, H. C.; Choi, S. H.; Kim, K. M.; Kim, J. H.; Park, K. S.; Jang, H. C.; Lim, $\mathrm{S}$. The effect of lithospermic acid, an antioxidant, on development of diabetic retinopathy in spontaneously obese diabetic rats. PLoS One 2014, 9, No. e98232.

(28) Huang, C.; Zheng, B. W.; Yu, W.; Niu, T. S.; Xiao, T. T.; Zhang, J.; Xiao, Y. H. Antibacterial effect evaluation of moxalactam against extended-spectrum beta-lactamase-producing Escherichia coli and Klebsiella pneumoniae with in vitro pharmacokinetics/pharmacodynamics simulation. Infect. Drug Resist. 2018, 11, 103-112.

(29) Huang, Q. F.; Zhang, S. J.; Zheng, L.; Liao, M.; He, M.; Huang, R. B.; Zhuo, L.; Lin, X. Protective effect of isoorientin-2 "-O-alpha-Larabinopyranosyl isolated from Gypsophila elegans on alcohol induced hepatic fibrosis in rats. Food Chem. Toxicol. 2012, 50, 1992-2001.

(30) Varadaraju, T. G.; Hwu, J. R. Synthesis of anti-HIV lithospermic acid by two diverse strategies. Org. Biomol. Chem. 2012, 10, 5456-5465.

(31) Escalante, A.; Gattuso, M.; Pérez, P.; Zacchino, S. Evidence for the mechanism of action of the antifungal phytolaccoside $\mathrm{B}$ isolated from phytolacca tetramera hauman. J. Nat. Prod. 2008, 71, 17201725.
(32) Wozniak, D.; Janda, B.; Kapusta, I.; Oleszek, W.; Matkowski, A. Antimutagenic and anti-oxidant activities of isoflavonoids from Belamcanda chinensis (L.) DC. Mutat. Res., Genet. Toxicol. Environ. Mutagen. 2010, 696, 148-153.

(33) Jang, H.-Y.; Ahn, K.-S.; Park, M.-J.; Kwon, O.-K.; Lee, H.-K.; Oh, S.-R. Skullcapflavone II inhibits ovalbumin-induced airway inflammation in a mouse model of asthma. Int. Immunopharmacol. 2012, 12, 666-674.

(34) Hyun, J. W.; Chung, H. S. Cyanidin and malvidin from Oryza sativa cv. Heugjinjubyeo mediate cytotoxicity against human monocytic leukemia cells by arrest of $\mathrm{G}(2) / \mathrm{M}$ phase and induction of apoptosis. J. Agric. Food Chem. 2004, 52, 2213-2217.

(35) Chan, K. W. K.; Ho, W. S. Anti-oxidative and hepatoprotective effects of lithospermic acid against carbon tetrachloride-induced liver oxidative damage in vitro and in vivo. Oncol. Rep. 2015, 34, 673-680.

(36) Umetrics, A. B. User's Guide to SIMCA-P, SIMCA-P+ version 11.0; Umetrics AB: UmeÅ, Sweden, 2005.

(37) Gollop, R.; Even, S.; Colova-Tsolova, V.; Perl, A. Expression of the grape dihydroflavonol reductase gene and analysis of its promoter region. J. Exp. Bot. 2002, 53, 1397-1409.

(38) Pasqua, G.; Monacelli, B.; Mulinacci, N.; Rinaldi, S. Giaccherini, C.; Innocenti, M.; Vinceri, F. F. The effect of growth regulators and sucrose on anthocyanin production in Camptotheca acuminata cell cultures. Plant Physiol. Biochem. 2005, 43, 293-298.

(39) Li, Y.; Van den Ende, W.; Rolland, F. Sucrose induction of anthocyanin biosynthesis Is bediated by DELLA. Mol. Plant 2014, 7, $570-572$.

(40) Payyavula, R. S.; Singh, R. K.; Navarre, D. A. Transcription factors, sucrose, and sucrose metabolic genes interact to regulate potato phenylpropanoid metabolism. J. Exp. Bot. 2013, 64, 51155131.

(41) Li, X.; Park, N. I.; Park, C. H.; Kim, S. G.; Lee, S. Y.; Park, S. U. Influence of sucrose on rutin content and flavonoid biosynthetic gene expression in seedlings of common buckwheat (Fagopyrum esculentum Moench). Plant Omics 2011, 4, 215-219.

(42) Nakabayashi, R.; Yonekura-Sakakibara, K.; Urano, K.; Suzuki, M.; Yamada, Y.; Nishizawa, T.; Matsuda, F.; Kojima, M.; Sakakibara, H.; Shinozaki, K.; Michael, A. J.; Tohge, T.; Yamazaki, M.; Saito, K. Enhancement of oxidative and drought tolerance in Arabidopsis by overaccumulation of antioxidant flavonoids. Plant J. 2014, 77, 367379.

(43) Lee, T. S.; Ho, Y. S.; Yeo, H. C.; Lin, J. P. Y.; Lee, D.-Y. Precursor mass prediction by clustering ionization products in LCMS-based metabolomics. Metabolomics 2013, 9, 1301-1310.

(44) Huan, T.; Forsberg, E. M.; Rinehart, D.; Johnson, C. H.; Ivanisevic, J.; Benton, H. P.; Fang, M.; Aisporna, A.; Hilmers, B.; Poole, F. L.; Thorgersen, M. P.; Adams, M. W. W.; Krantz, G.; Fields, M. W.; Robbins, P. D.; Niedernhofer, L. J.; Ideker, T.; Majumder, E. L.; Wall, J. D.; Rattray, N. J. W.; Goodacre, R.; Lairson, L. L.; Siuzdak, G. Systems biology guided by XCMS Online metabolomics. Nat. Methods 2017, 14, 461-462.

(45) Saeed, A. I.; Bhagabati, N. K.; Braisted, J. C.; Liang, W.; Sharov, V.; Howe, E. A.; Li, J.; Thiagarajan, M.; White, J. A.; Quackenbush, J. TM4 microarray software suite. Methods Enzymol. 2006, 411, 134193.

(46) Lee, D. Y.; Fiehn, O. High quality metabolomic data for Chlamydomonas reinhardtii. Plant Methods 2008, 4, 7. 